\title{
The New Avalanche-Like Stochastic Model for Parameterization of Seismicity and Its Application to the South Sakhalin Island Seismicity
}

\author{
M. V. Rodkin ${ }^{1,2}$ and I. N. Tikhonov ${ }^{2}$ \\ ${ }^{1}$ Institute of Earthquake Prediction Theory and Mathematical Geophysics, Russian Academy of Sciences (RAS), Moscow 117997, Russia \\ ${ }^{2}$ Institute of Marine Geology \& Geophysics, Russian Academy of Sciences Far Eastern Branch (FEB RAS), \\ Yuzhno-Sakhalinsk 693022, Russia
}

Correspondence should be addressed to I. N. Tikhonov, tikhonov@imgg.ru

Received 9 August 2011; Revised 25 December 2011; Accepted 18 January 2012

Academic Editor: Yamaoka Koshun

Copyright ( $) 2012$ M. V. Rodkin and I. N. Tikhonov. This is an open access article distributed under the Creative Commons Attribution License, which permits unrestricted use, distribution, and reproduction in any medium, provided the original work is properly cited.

\begin{abstract}
Seismic process is usually considered as an example of occurrence of the regime of self-organizing criticality (SOC). A model of seismic regime as an assemblage of randomly developing episodes of avalanche-like relaxation, occurring at a set of metastable subsystems, can be the alternative of such consideration. The model is defined by two parameters characterizing the scaling hierarchical structure of the geophysical medium and the degree of metastability of subsystems of this medium. In the assemblage, these two parameters define a model $b$-value. An advantage of such approach consists in a clear physical sense of parameters of the model. The application of the model for parameterization of the seismic regime of the south part of Sakhalin Island is considered. The models of space changeability of the scaling parameter and of temporal changeability of the parameter of metastability are constructed. The anomalous increase of the parameter of metastability was found in connection with the Gornozavodsk and Nevelsk earthquakes. At the present time, high values of this parameter occur in the area of the Poyasok Isthmus. This finding is examined in comparison with other indications of an increase in probability of occurrence of a strong earthquake in the South Sakhalin region.
\end{abstract}

\section{Introduction}

Seismic process is usually considered as an example of realization of the self-organized criticality-the SOC-model [1-3]. However, as it was argued in [4] the SOC model has a rather limited possibility in interpretation of real seismotectonic processes. Besides, there is no clear interpretation in terms of this model of a difference between regions of high and low seismic activity. Moreover, the analogue between critical phenomena and seismic process is not satisfying enough. The critical phenomena (the second-order phase transitions, for example) proceed without discharge or absorption of energy; and this is their fundamental peculiarity, in many respects determining other features of the critical behavior. But earthquakes are accompanied by release of huge amounts of energy, and this is their fundamental pro- perty. Thus it can be concluded, that consideration of a seismic process in terms of the SOC-model is not quite satisfactory. Therefore, alternative approaches are of interest.

For quantitative statistic modeling of seismicity regime, the Generalized Omori law and the Epidemic-Type Aftershock-Sequence (ETAS) model are used at the present time [5-7]. However, these models have a formal statistical character; the determination of parameters of the models and even the findings of statistical relations between the parameter values do not result in an essential progress in understanding of the physics of the seismic process.

The fundamental properties determining the process of seismicity are the scaling hierarchical properties of the structure of the Earth's crust and the irreversibility of the processes ongoing in the Earth's interior. A natural model for understanding of the process of seismicity would be a statistical 
model treating seismicity in terms of these fundamental characteristics. The model of seismic process as an assemblage of avalanche-like episodes of relaxation, occurring occasionally at a set of uniform metastable subsystems [8, 9], meets such demands. As it was shown in [8], this model appears to be the most simple, providing the realization of power law distributions typical of dynamical dissipative systems [10]. In case of seismicity, metastability is connected with elastic energy stored in the geophysical media and abruptly released during earthquakes. In application to the earthquake process, we will name this model the statistical earthquake model (SEM). The main parameters of the SEM model are two parameters, characterizing the scaling properties of the geophysical media and the level of irreversibility (metastability) of processes taking place in this media. The SEM model, which was discussed in detail in [9], is presented and used below in the examination of the seismic regime of the south part of Sakhalin Island.

\section{Model}

We will model seismic regime as an assemblage of episodes of avalanche-like relaxation, occurring occasionally at a set of statistically identical metastable subsystems. Let us describe this statistical earthquake model (SEM) in terms of recurrent scheme (or equivalently in terms of multiplicative process) as it was presented in $[8,9]$. A longer description of a continuous case is presented in [11].

Let us imagine that an ongoing stochastic process (here earthquake), that had released energy $X_{i}$ by time moment $t_{i}$, continues its development with probability $p$ or cancels with probability $(1-p)$. In a case when the process interrupts on this ith step, the quantity of event (the amount of energy released in the event) will be equal to $X_{i}$. In a case when the process of relaxation of metastable subsystem continues, we suggest that the energy released in this event will grow up by the next moment of time $t_{i+1}$ to the value

$$
X_{i+1}=r \times X_{i}
$$

where $r$ can be a random parameter with mean value exceeding one. In a continuous case [11], the avalanche-like differential equation instead of recurrent relation (1) is used. This approach models an avalanche-like process of release of metastable systems.

For simplicity of the mathematical manipulations presented below, we will suggest that the constant $X_{0}$ value at the first step being equal to $X_{0}=1$ and $r=$ const. In this case, in scheme (1) the probability of interrupting of the process on $n$th stage and correspondingly obtaining of the value $X_{n}=r^{n}$ is equal to $(1-p) \times p^{n}$. From this, we observe that the tail of the function of distribution $F\left(X_{n}>X\right)$ is equal to

$$
\begin{aligned}
(1-F(X)) & =(1-p) \times p^{n} \times\left(1+p+p^{2}+\cdots p^{\propto}\right) \\
& =p^{n} .
\end{aligned}
$$

We have also $\lg (X)=n \times \lg (r)$, and thus $n=\lg (X) / \lg (r)$ and $\lg (1-F(X))=n \times \lg (p)=\lg (X) / \lg (r) \times \lg (p)$. From here, we have

$$
(1-F(X))=X^{\lg (p) / \lg (r)}
$$

thus we receive a power law dependence for the tail of the distribution function $(1-F(X))$ from $X$, as it takes place in the distribution law of the seismic moment and seismic energy values and in many other cases [12]. It can be shown that this result is valid for the case of stochastic $r$ values (for mean $r$ value $>1$ ) and in case of random normal distribution of $X_{0}$ values that has a minor influence on the final type of distribution (3).

The scheme thus described treats a development of an earthquake as a process of sequential transition to higher hierarchical levels. At constant parameter $r$ value, we have a discrete and log-periodical distribution of the energy values of earthquakes. With a growth of random spread in $r$ values, the step-by-step character of model distributions becomes smoother, and at the limit we receive a monotonic distribution with quasirectilinear relation in coordinates $\{\lg (X), \lg (1-F(X))\}$ with a slope of recurrence relation equal to

$$
b=-\frac{\lg (p)}{\lg (r)}
$$

where $b$ characterizes the power distribution of quantities $X$ and has a meaning similar to the $b$-value in the GutenbergRichter law (for the energy or seismic moment earthquake values). The sign minus is added in (4) to get a positive $b$ value used in seismology.

Thus, in terms of the SEM model, the $b$-value is defined by two parameters, one of them $(r)$ characterizes scaling properties of the medium, whereas the second $(p)$ answers a probability of a continuation of avalanche-like relaxation of metastable sub-systems. Thus it characterizes the degree of metastability of the medium. We will name these two parameters further as a scaling parameter $r$ and a metastability parameter $p$.

It is not difficult to pick up the values of $r$ and $p$ parameters of the SEM model and initial $X_{0}$ value so that the received $b$ parameter from (4) will agree with $b$-value of a typical seismic regime, and $\lg (X)$ will have values typical of earthquake magnitudes. If we take some average number $N$ of avalanche-like processes occurring in a time unit, and the suitable $r(t)$ and $p(t)$ values, the model will give the sequence of magnitudes of main (independent) events $\lg \left(X_{j}\right)$ similar with a sequence of magnitudes of earthquakes (without aftershocks) occurring in a real seismic process.

As an example, we take a case with weak (with amplitude 0.2 ) and periodic ( $T=1000$ time units) change of parameter $p$ producing the similar periodicity in a model $b$-values. The mean intensity of seismic flow $N=500$ events per unit time is suggested, and variations in $N$ number are assumed to follow the Poisson law. In Figure 1, an example of such model process of duration of 5000 time units is presented. For the every one time unit, the model maximal magnitude $M_{\max }$ 

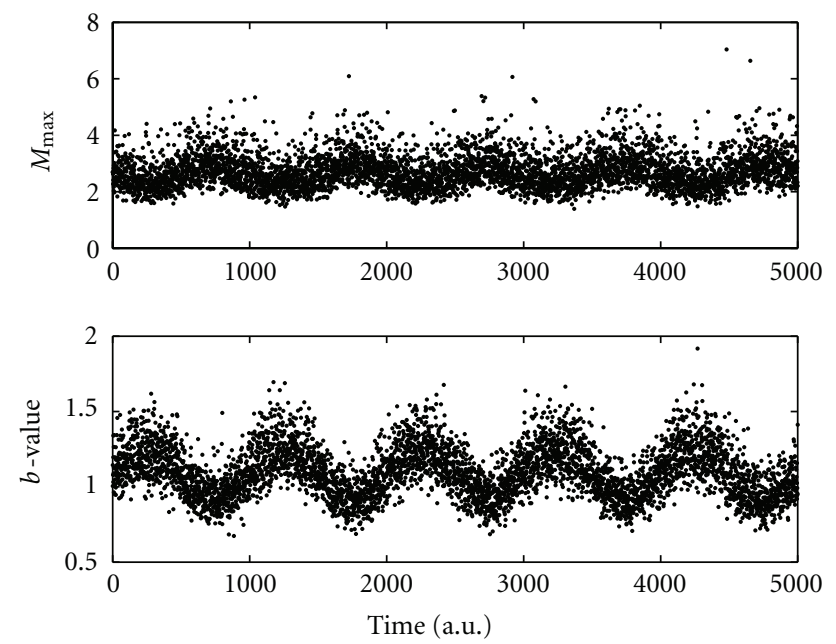

FIGURE 1: An example of realization of the SEM model of a seismic regime; (a) maximal magnitude values $M_{\max }$; (b) the $b$-values. On an abscissa axis, the arbitrary time units are given (500 events in average in each).

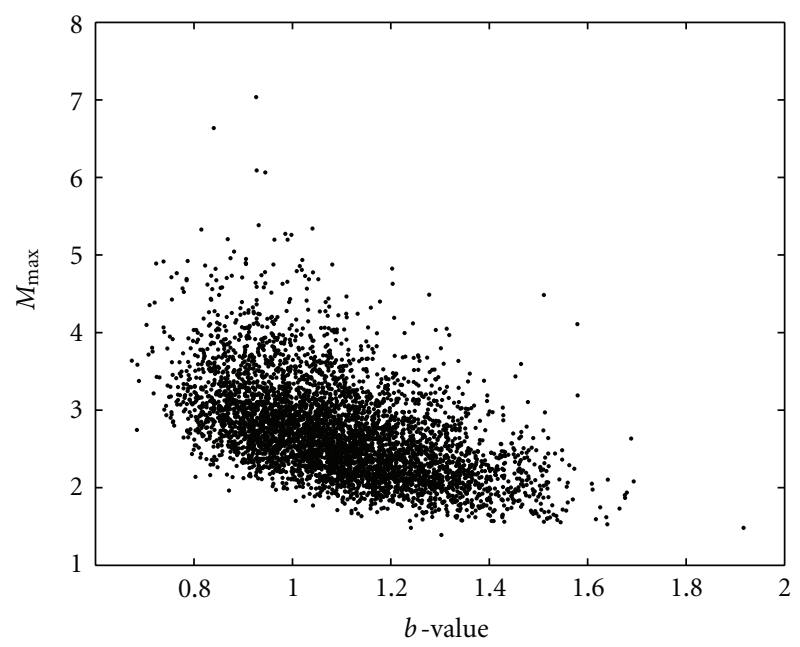

Figure 2: Model relationship between the $b$-value and maximal magnitude $M_{\max }$ value occurred in the next time interval.

value and the $b$-value are calculated. The $b$-values are calculated for the every one time unit from the maximum likelihood approach [13] and the model magnitude values $\lg \left(X_{i}\right)$. The received $M_{\max }$ and the $b$-values appear to be similar visually with typical behavior of a real seismic process (besides of an artificially taken periodical character of change in $b$-values with time).

It is easy to see that even such a very simple model is not trivial. It produces the well-known "prognostic" featurethe decrease in $b$-values precedes the intervals of time of occurrence of strong earthquakes. To see such dependence (distinct also in Figure 1) more clearly, in Figure 2 the graph of relationship of maximal magnitudes $M_{\max }=\lg \left(X_{i}\right)$ values versus $b$-values in preceding time interval was shown.

An appearance of model correlation given in Figure 2 is clear. Actually, the values of $p$ and $r$ parameters that cor- respond statistically to occurrence of larger $M_{\max }$ values correspond also to lesser $b$-values. This relation has a stochastic character. It is worth mentioning that the decrease of $b$ values in the SEM model is not an indicator of developing of process of a "preparation" of a strong event (it is not correct to speak about the preparation of a "strong earthquake" in the case of a sequence of independent events), but it is a parameter correlated with an increase of probability of occurrence of a strong event.

Thus the increase of the values of parameters $p$ and $r$ is an indicator of increase of probability of occurrence of a strong earthquake, and so the evaluation of these parameters and their changeability can be used for monitoring of a probability of a strong earthquake occurrence. In the SEM model, it appears naturally (but not obligatory) to consider the scaling parameter $r$ as depending on the Earth's crust segmentation (so parameter $r$ is spatially dependent and constant or slow changeable through time), whereas the parameter metastability $p$ is suggested to be time dependent.

The SEM model is used below for examination of the seismic regime of the south of Sakhalin Island. However, before discussing the results of such examination, we should briefly characterize the seismicity of the Sakhalin Island and the used database.

\section{Patterns of Seismicity of the Sakhalin Island and the Available Earthquake Catalogs}

Sakhalin Island (Russia) is located in the Pacific-Eurasia transition zone. In the island, on average 1 earthquake with magnitude $M \geq 6$ and about 10 events with $M \geq 5$ takes place every 10 years. The events with $M \geq 7$ occurred nearly once per century. The strongest known Moneron earthquake M 7.5 took place here in 1971 .

The seismicity of Sakhalin can be divided into shallow $(h=0-30 \mathrm{~km})$ and deep (mainly in depth interval of 280$350 \mathrm{~km}$ ) seismicity. Deep earthquakes are connected with the Kurile Islands subduction zone. In Sakhalin Island, deepfocus earthquakes do not represent a substantial seismic danger, and shallow seismicity appears to be not dependent on deep seismicity. Below, only shallow earthquakes with the depth $h<30 \mathrm{~km}$ are considered.

Within Sakhalin Island and the adjacent shelf, four major deep fault systems were identified that generate almost all crustal earthquakes with $M \geq 5$.5: the Rebun-Moneron, the Western Sakhalin, the Central Sakhalin, and the Eastern-Sakhalin fault systems (Figure 3).

The Rebun-Moneron fault system, situated near the Moneron and Rebun Islands, was revealed to be active when the century's strongest shallow-focus Sakhalin earthquake $\left(M_{S}\right.$ 7.5, September 5, 1971) took place here.

The Western Sakhalin fault system extends below the floor of the Tatar Straight along the western shore of the island and then merges with the Central Sakhalin faults. In this zone strong earthquakes in 1907 (Alexandrovsk-Sakhalinsk, $M_{S}$ 6.5), 1924 (Lesogorsk-Uglegorsk, $M_{S}$ 6.9), and in 2000 (Uglegorsk, $M_{S}$ 7.2) took place. No strong earthquakes $(M>5.0)$ were registered in the southern part of this fault zone until 


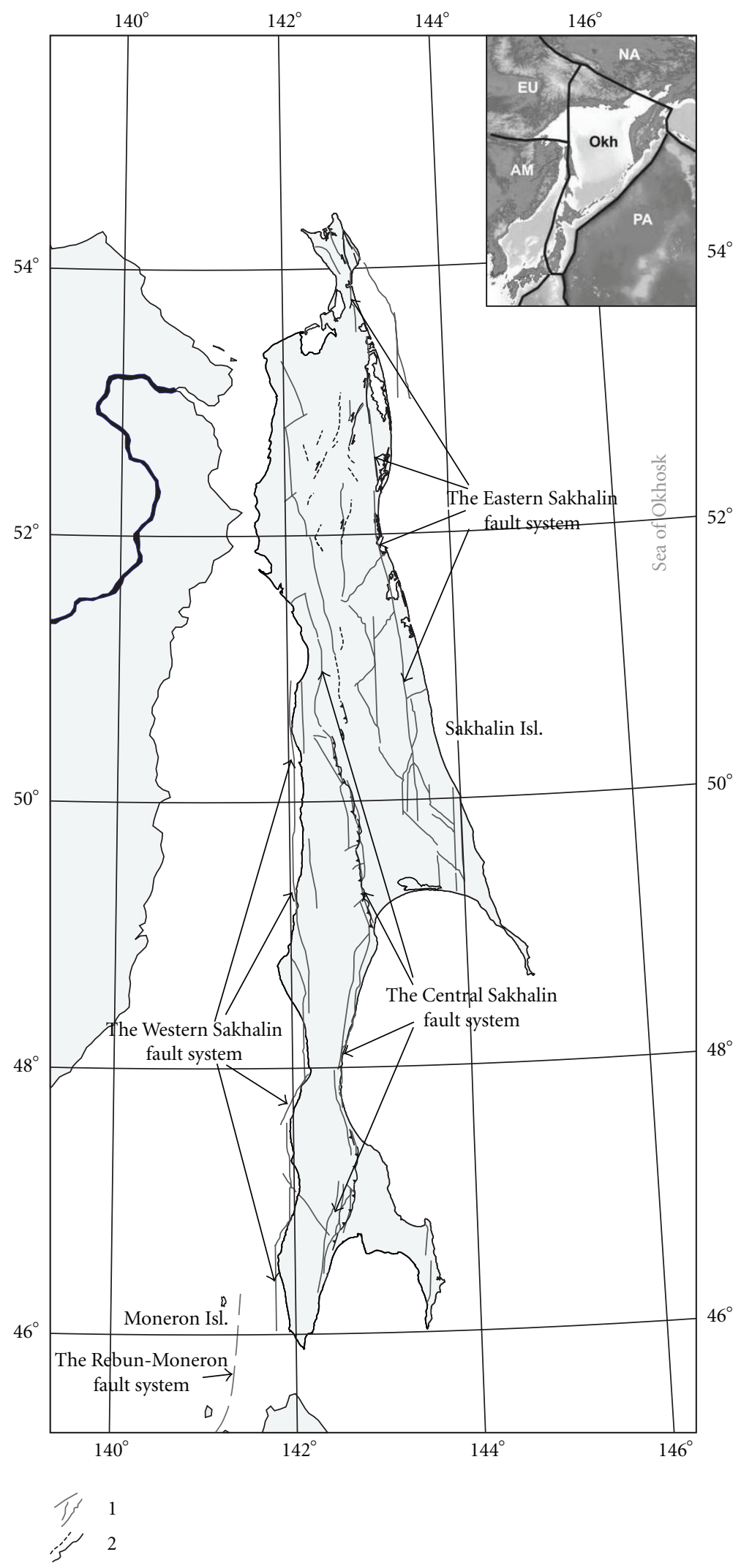

FIgURE 3: Active faults of the Sakhalin region. Insert-regional scheme of plate boundaries in the model NUVEL-1A and its modifications [14]. NA-North American, EU-Eurasian, PA—Pacific, OKH-Okhotsk plates. 
the 17 August 2006, $M_{W}$ 5.6 Gornozavodsk earthquake and the 2 August 2007, $M_{W} 6.2$ Nevelsk earthquake have occurred here $[15,16]$.

The Central Sakhalin fault system, and more specifically, its southern segment, is traceable from south to north along the western coast of Aniva Bay (Krilion Peninsula), farther on westward from Yuzhno-Sakhalinsk, and along the eastern shore of the island to Poronaisk, and then merges with the Western Sakhalin faults. Two strongest earthquakes that occured in this fault zone since 1905 (February 2, 1951 Aniva earthquake $\left(M_{S}\right.$ 5.5) and the September 1, 2001 Takoe earthquake $\left.\left(M_{S} 5.6\right)\right)$ took place within the southern segment of the fault.

The Eastern Sakhalin fault system extends along the north-eastern shore of the island. Prior to the May 28, 1995 Neftegorsk earthquake $\left(M_{S} 7.2\right)$, there was no evidence of a significant earthquake occurrence here. Investigation of active faults of the North Sakhalin began after the Neftegorsk earthquake [17-19]. Paleoseismological reconstructions showed that recurrence time of the strong ( $M$ 7.0-7.5) earthquake here appears to be from some hundreds to thousand years.

The most complete data about the Sakhalin Island earthquakes during the historical and instrumental periods of observations are collected in the regional catalogue [20] (see Figure 4). The catalogue is unified, and all earthquakes are characterized in $M_{L H}$ magnitude scale. 3566 events with magnitudes $M_{L H} \geq 3.0$ occurring in 1905-2005 are given in the catalog. The representativeness of the catalog changes considerably with time. The catalog is believed to be representative (the Gutenberg-Richter frequency-magnitude relation is fulfilled) for the events with $M_{L H} \geq 5.5$ since 1930, and for the events with $M_{L H} \geq 3.5$ since 1970. This catalog named below as catalog 1 is used for the examination of spatial change of the scaling parameter $r$ of the SEM model.

Let us consider the main features of the spatial distribution of epicenters with $M_{L H} \geq 3.5$ (Figure 4). As it is seen in the figure, three areas with higher seismicity are observed in the Sakhalin Island and the adjoining shelf: (1) western part of the Southern Sakhalin with adjacent shelf southward $47.0^{\circ} \mathrm{N}$; (2) western and Central parts of the Central Sakhalin with adjacent shelf between $48.5^{\circ}$ and $51.5^{\circ} \mathrm{N}$; (3) eastern part of the Northern Sakhalin with adjacent north-eastern shelf northward $51.5^{\circ} \mathrm{N}$. All the earthquakes with $M_{L H} \geq 5.5$ occurred within these three areas; in these regions the most part of the shocks with $M_{L H} \geq 4.5$ have also occurred.

It can be noticed that localization of the strong earthquakes appears to agree with a suggested location of the boundary of Okhotsk Sea plate in this region. It is suggested [21] that this boundary in the south goes along the western shore of the island up to the latitude $51^{\circ} \mathrm{N}$, then it turns to the east and crosses the island along the valley of Tym River and extends further northward along the eastern shore of the island. There are, however, a number of gaps in localization of strong seismicity along this tentative plate boundary zone. Below, the most southern gap in strong seismicity taking place along the western shore of the Sakhalin Island between $47.0^{\circ} \mathrm{N}$ and $49^{\circ} \mathrm{N}$ neighboring to the location of the $M_{W} 6.2$ Nevelsk and $M_{S} 7.5$ Moneron earthquakes (Figure 5) will be examined. This site was argued earlier [22] as a seismic gapa potential area of the origin of a next strong earthquake [23].

More detailed information about the seismicity of the South Sakhalin area is available since 2003 because of the installation of the seismic networks "Datamark" and "DAT." The catalog obtained from these networks is presented in unified $M_{L}$ magnitude scale. In the latest version of this catalog published last year [24], which was used, it is argued that the network provides the registration of $M \geq 2.5$ earthquakes throughout the South Sakhalin and adjacent shelf area and $M \geq 2.0$ earthquakes in the central part of the South Sakhalin area. The Gutenberg-Richter frequencymagnitude relation of the catalog data is found to be valid for the earthquakes with $M \geq 2.0$. This catalog named below as catalog 2 is used below for the examination of the spatial change of the parameter of metastability $p$ of the SEM model. The Gutenberg-Richter relations for both catalogs 1 and 2 are presented in Figure 6. This Figure shows the suitable representativeness of earthquakes with magnitude $M \geq 3.5$ and $M \geq 2.0$ for catalog 1 and catalog 2 correspondingly.

\section{Parameterization of Seismicity of the South of Sakhalin in the Framework of the SEM Model}

We have used the catalogue 1 [20] and its continuation to examine the spatial model of change of the scaling parameter $r$. Firstly, the $b$-values were estimated in the surroundings of every event of the used catalogue. Estimation of the $b$-value was performed for the groups including 50 events closest to the given earthquake. For the $b$-values estimation the maximum likelihood method was used [13]:

$$
b=\frac{\lg (e)}{M_{\mathrm{av}}-M_{c}},
$$

where $e=2.7183 \cdots, M_{\mathrm{av}}$ is the average magnitude for the given subset of data and $M_{c}$ is the lower magnitude limit. Estimates from (5) are known to be suitably stable for number of events exceeding 50 . Then by formula (4) the value of parameter $r_{i}$ for spatial surrounding of the $i$ th earthquake was estimated. At this step, the value of probability $p$ was taken to be fixed $p=0.5$. For the further use, the scaling parameter $r_{i}$ values were spatially averaged in cells $1 / 3^{\circ}$ of latitude $\times 1 / 3^{\circ}$ of longitude to obtain $R(\varphi, \lambda)$ values. This way we have got $R(\varphi, \lambda)$ values as spatially averaged scaling parameter $r$ values.

Having in mind the change in representativeness of the catalog 1 through time the different variants of time interval and magnitude limitation were used for $r_{i}$ and $R(\varphi, \lambda)$ calculation. One of the received $R(\varphi, \lambda)$ models obtained for the case of $M_{L H}>3.5$ earthquakes occurring since 1970 (there are 1224 such events in the catalog 1) is shown in Figure 7. In all examined cases of different time intervals and limitation of magnitude range, the main features of the $R(\varphi, \lambda)$ map are similar. We have considerable increase in $R(\varphi, \lambda)$ values in the area adjacent to the location of occurrence of the strongest Moneron and Nevelsk earthquakes, and a slight tendency of a decrease in scaling parameter value from south to north 


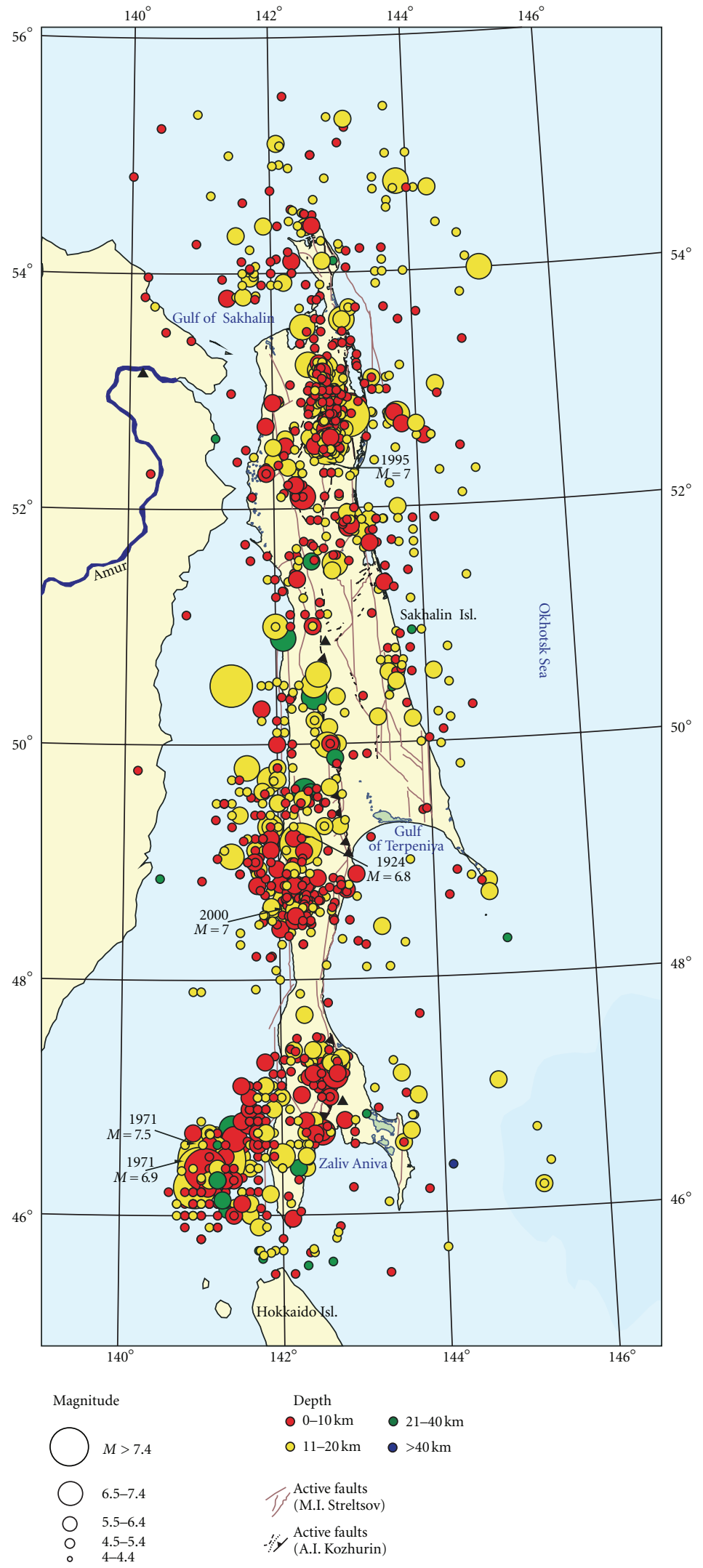

FIGURE 4: Map of crustal $M_{S} \geq 3.5$ earthquake of the Sakhalin region, 1906-2010. Date and magnitude is given for the strongest earthquakes. 


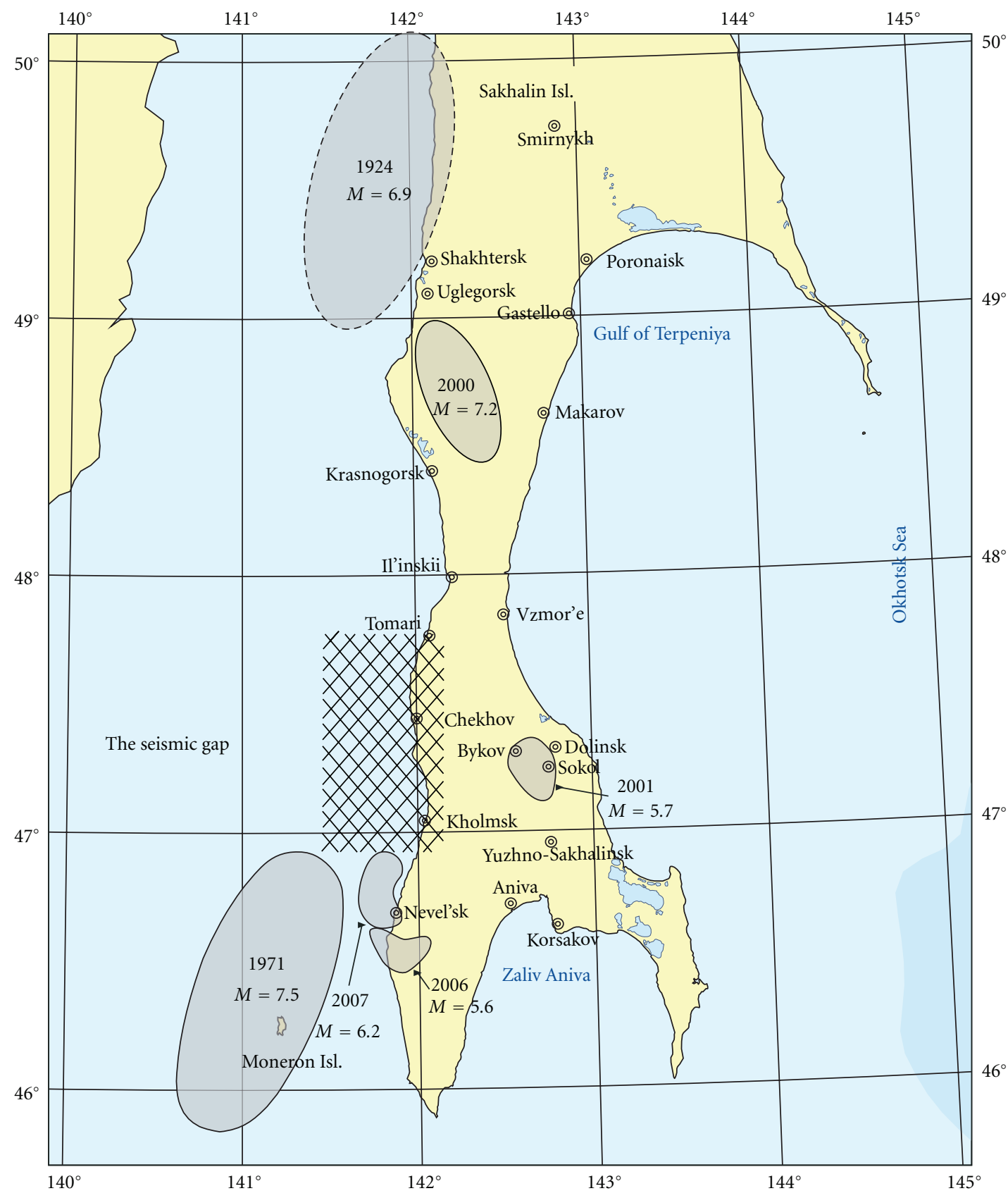

FIGURE 5: Sources of large earthquakes at the western coast of Sakhalin Island (grey ovals) and the approximate location of the seismic gap (hatched rectangle).

in the South Sakhalin region. Note also that the range of change of $R(\varphi, \lambda)$ values is slightly larger than it was expected. This can occur due to changeability of other factors factually included at this step in change of $R(\varphi, \lambda)$ value (remember, that parameter $p$ was suggested to be constant, $p=0.5$ ).

For earthquake prediction, however, the time changes of probability $p$ are of the main interest. In estimation of temporary changeability of parameter of metastability $p$, the errors in determination of $R(\varphi, \lambda)$ values do not play an essential role, because the values $R(\varphi, \lambda)$ are suggested to be constant in time and so the errors of their determination have a minor importance in examination of change in parameter metastability $p$ values with time.

The estimation of time changeability of parameter of metastability $p$ was carried out using the detailed catalogue 2 obtained from the networks "Datamark" and "DAT" and the obtained before model of spatial changeability of scaling parameter, that is, from $R(\varphi, \lambda)$ values. As above, in the case of the $R(\varphi, \lambda)$ values determination different variants of time and magnitude intervals were examined. In the version presented below, we have examined earthquakes with $M \geq 2.5,1789$ events altogether. Firstly, we calculated local 


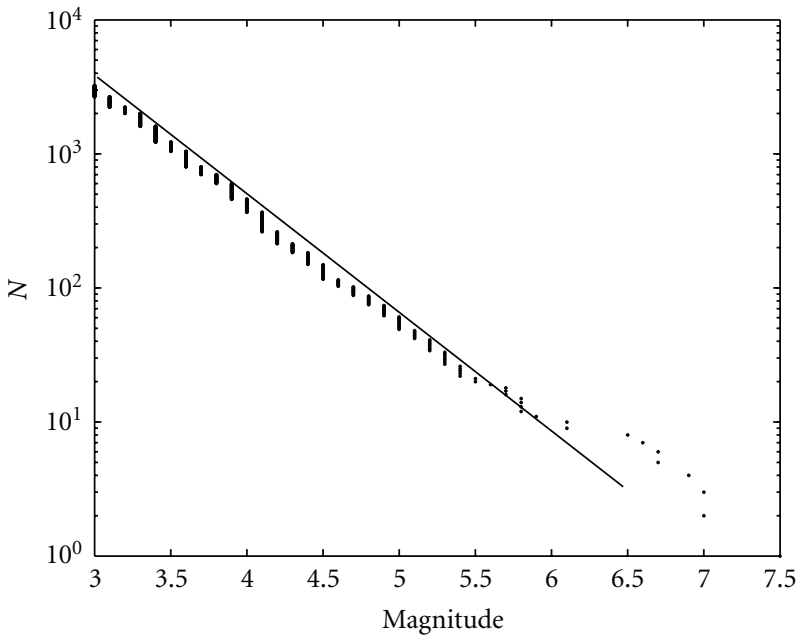

(a)

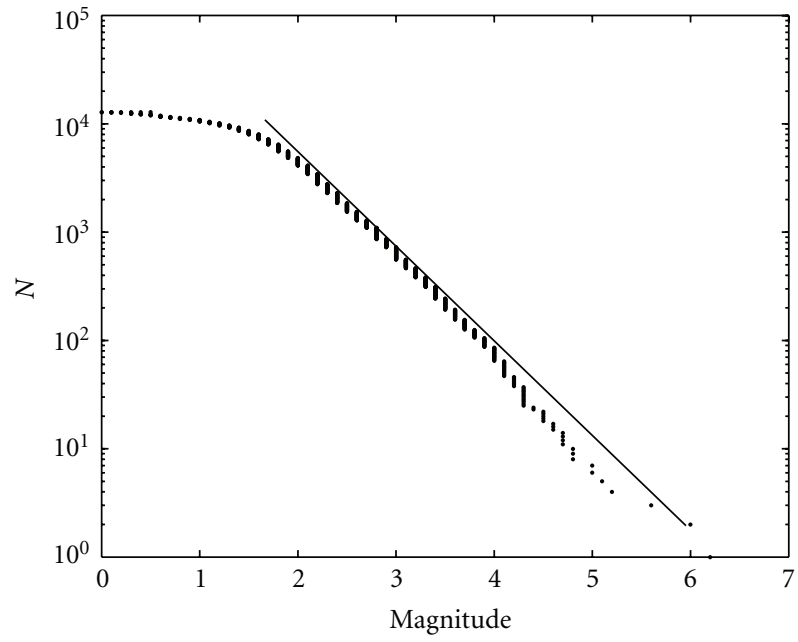

(b)

FIgURE 6: Gutenberg-Richter relations for both catalogs 1(a) and 2 (b); points—earthquakes of magnitude $M$ in descending order, line shows the limits of validity of Gutenberg-Richter relation.

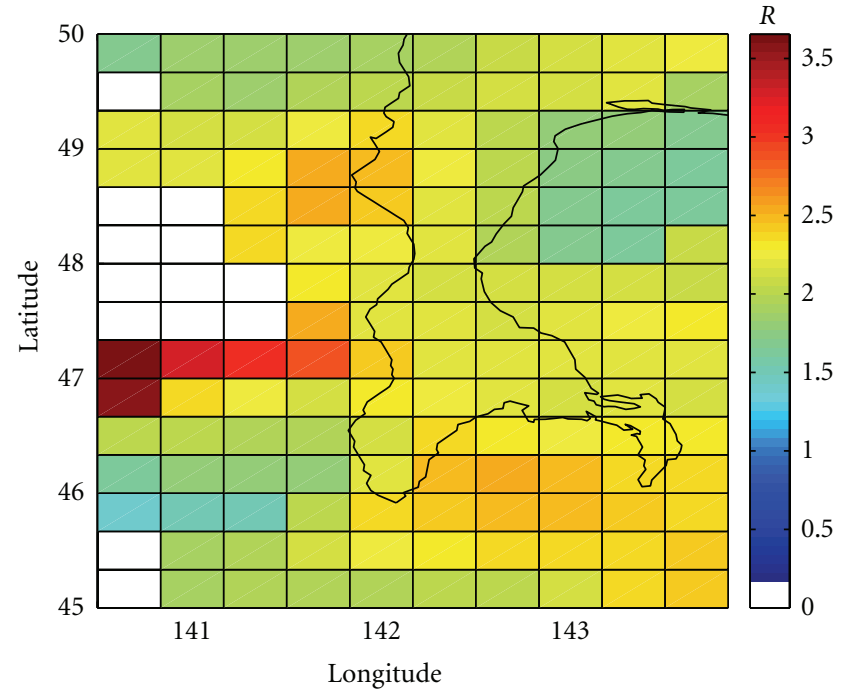

Figure 7: Scheme of changes of the values $R(\varphi, \lambda)$, spatial component of the SEM model.

$b$-values for the spatial-temporal surrounding of every of these earthquakes. As above, 50 events spatially closest to every given $i$ th earthquake were chosen to estimate the corresponding $b$-value from relation (5); however, the selection was done not from all the assemblage of the epicenters, but only from a temporal subsequence of events from $(i-500)$ to $(i+500)$; the length of the sequence decreases for the events adjacent to the ends of the temporary area. The quantity of parameter $p_{i}$ was estimated then from (4) with due account of the value of the scaling parameter $R(\varphi, \lambda)$ corresponding to given coordinates and the obtained time-local $b$-value.

In Figure 8 , the values $p(t)_{i}$ for every $i$ th event of catalogue 2 , obtained by the described method are shown. In Figure two temporary areas of high concentration of the earth-

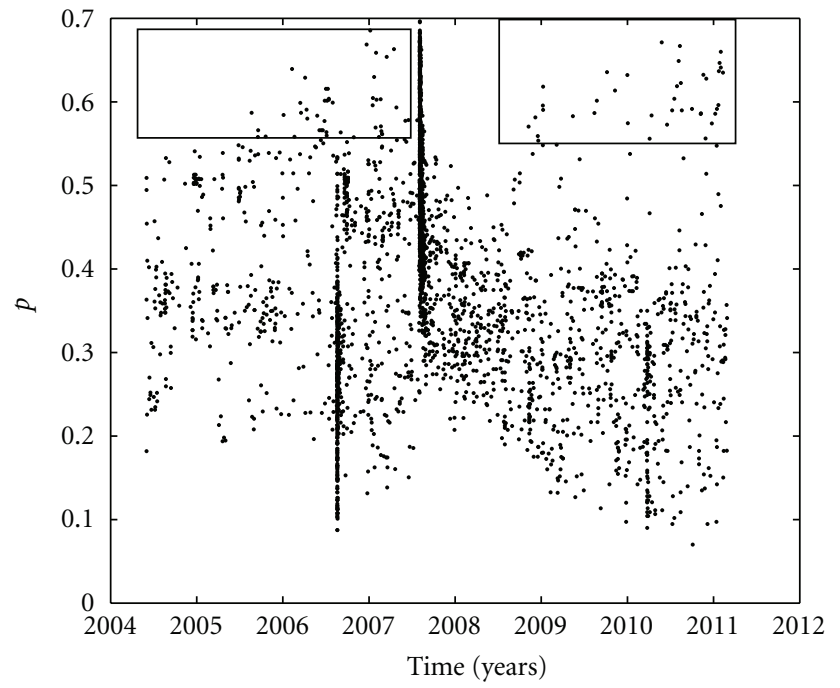

FIGURE 8: Temporary component of the SEM model-sequence of values of parameter of metastability $p$ in spatial-temporary surrounding of earthquakes of the catalogue 2 . The groups of the events with higher $p$ values ( $p>0.55)$, occurred before and after the Nevelsk earthquake, are marked out by the rectangles.

quakes, corresponding to the Gornozavodsk (17 August 2006, $46.51^{\circ} \mathrm{N}$ and $141.92^{\circ} \mathrm{E}, M_{W}$ 5.6) and Nevelsk (2 August, $2007,46.83^{\circ} \mathrm{N}$ and $141.76^{\circ} \mathrm{E}, M_{W} 6.2$ ) earthquakes, are well seen. Some tendency of an increase in parameter $p$ values before the Nevelsk earthquake, and a clear tendency of decrease after the Nevelsk earthquake occurrence can be seen. This tendency is fairly valid. In terms of the SEM model, this feature points out the growth of probability of occurrence of a strong earthquake that has realized in the Nevelsk earthquake occurrence. On this background the groups of events with anomalously high $(>0.55)$ parameter $p$ values are highlighted. The first such group takes place before the Nevelsk 


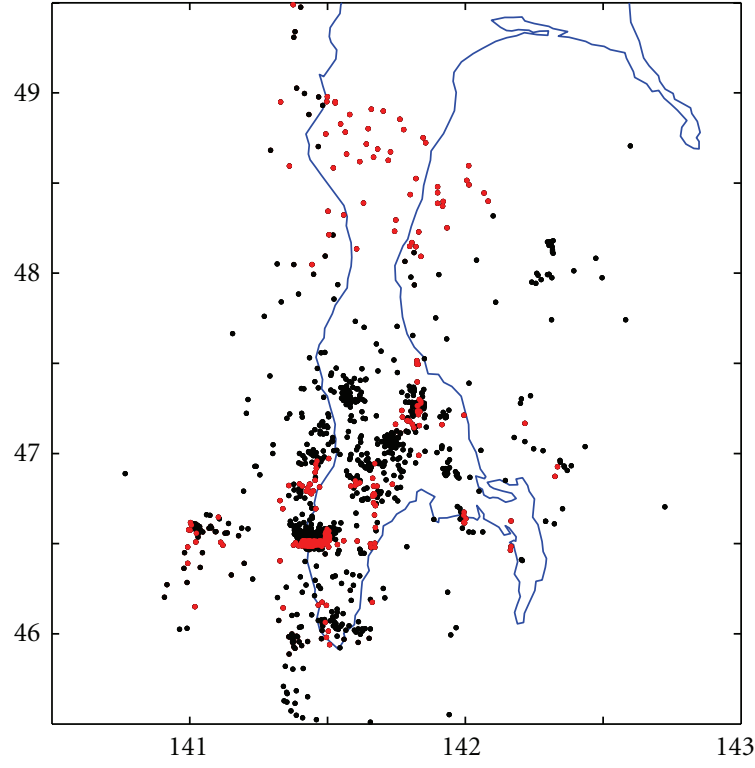

(a)

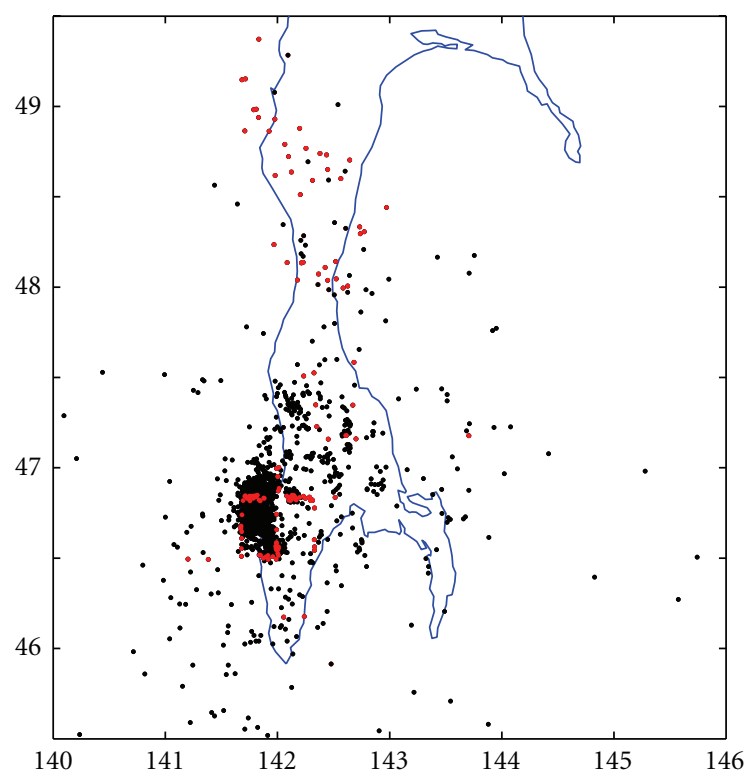

(b)

Figure 9: Location of earthquakes with typical $(p<0.55$, black points) and with increased values of parameter of metastability $(p>$ 0.55 , red points). (a) events occurred before the Nevelsk earthquake; (b) events occurred after the Nevelsk earthquake.

earthquake, and the second group begins in one year after the Nevelsk earthquake occurrence and prolongs till now. These two groups are highlighted in Figure 8 by the rectangles. In Figures 9(a) and 9(b), spatial location of epicenters of the examined events of the catalogue 2 occurring before and after the Nevelsk earthquake are given. In both cases, the events with metastability parameter $p$ values exceeding 0.55 are given as red points. We cannot explain some regularity in location of events with parameter $p$ values exceeding 0.55 , probably it can be connected with some quantization of latitude and longitude values in the catalog.
The groups of the epicenters with $p>0.55$, occurring before the Nevelsk earthquake took place in a number of locations connected with epicenters of strong earthquakes and earthquake swarms occurring in this time interval. Besides, during the same time interval events with higher values of parameter of metastability $p$ were found to be typical of the more distant area located in the Poyasok Isthmus region with latitude values in interval $48-49^{\circ} \mathrm{N}$.

The higher level of parameter of metastability revealed in the area of the Poyasok Isthmus can be connected probably with the Southern Sakhalin fault $[25,26]$, which represents a large transversal nonconformity across the Sakhalin Island. The greater activity of this structure before the Nevelsk earthquake could be explained probably by an analogy with the effect of activity of transverse structures in the straits of the Kuril Islands in connection with the strong earthquakes occurring at the adjacent segments of the subduction zone. Such analogy is substantiated by close correlation of the areas of strong earthquakes occurrence in the Sakhalin Island with the tentative location of the Okhotsk Sea plate boundary.

After the Nevelsk earthquake of August 2, 2007, the earthquakes with higher parameter $p$ value timely disappeared (Figure 8). However, such earthquakes arose again one year later. The events with $p>0.55$ took place in a few areas connected with the Nevelsk earthquake occurrence and in the Poyasok Isthmus area. Besides, a few events with high parameter metastability value are dispersed irregularly around the studied area; those can be caused by stochastic errors.

According to the catalog 1 [20], the Poyasok Isthmus area corresponds to the seismic gap between two segments of high seismic activity taking place in the last century, so it can be suggested that a considerable seismic activity could occur in this gap also. Having this possibility in mind we have examined the seismicity in the Poyasok Isthmus area in more detail. The growth of seismic danger is associated rather frequently with a nonlinear growth of a number of events and released seismic energy with time. To check this effect, the graphs of a number of events and released seismic energy in the zone (rectangle area $47.8^{\circ}-49^{\circ} \mathrm{N}$ and $141.5^{\circ}-144^{\circ} \mathrm{E}$ ) were calculated from the catalog 2 data. In Figure 10(a), the cumulative graph of a number of events occurring here since 2005 is given, and all earthquakes with magnitude exceeding 2 were taken into account to increase a statistics. In Figure 10(b), the graph of cumulated seismic energy released here since 2009 is shown (if we could show data for an earlier period of time, the changes in flow of seismic energy after 2009 would become badly visible).

In Figure 10(a), several intervals of nonlinear growth of a number of events are seen. These time intervals appear to be associated with the strongest earthquakes which occurred during this time interval in the south of Sakhalin Island. The moments of these earthquakes occurrence are marked by vertical lines and figures: 1-Nevelsk, 2-Gornozavodsk, and 3-the event of February 24, 2007 with $M_{S} 4.6$ and coordinates $48.95^{\circ} \mathrm{N}, 142.06^{\circ} \mathrm{E}$. The first two events are the strongest earthquakes occurring in the studied time interval in the south of Sakhalin. The third event is the strongest earthquake, which occurred in the Poyasok Isthmus region 


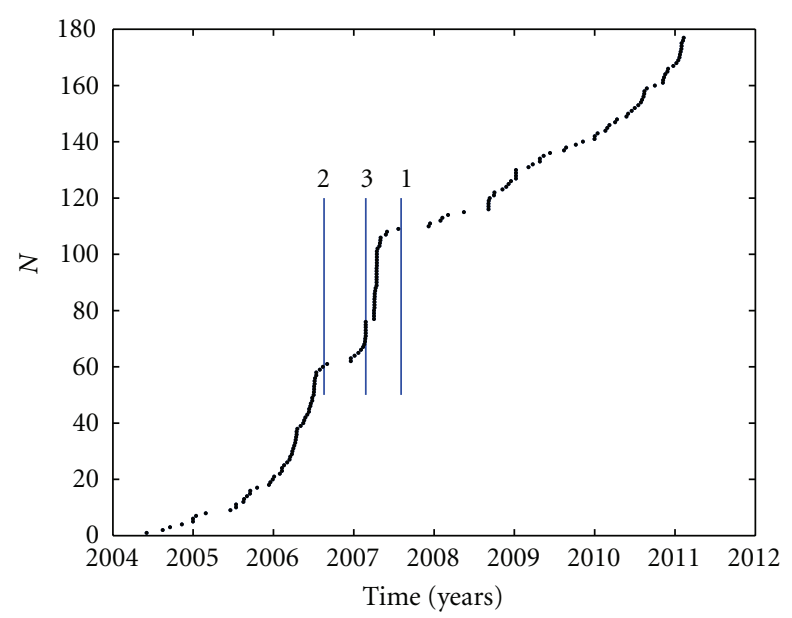

(a)

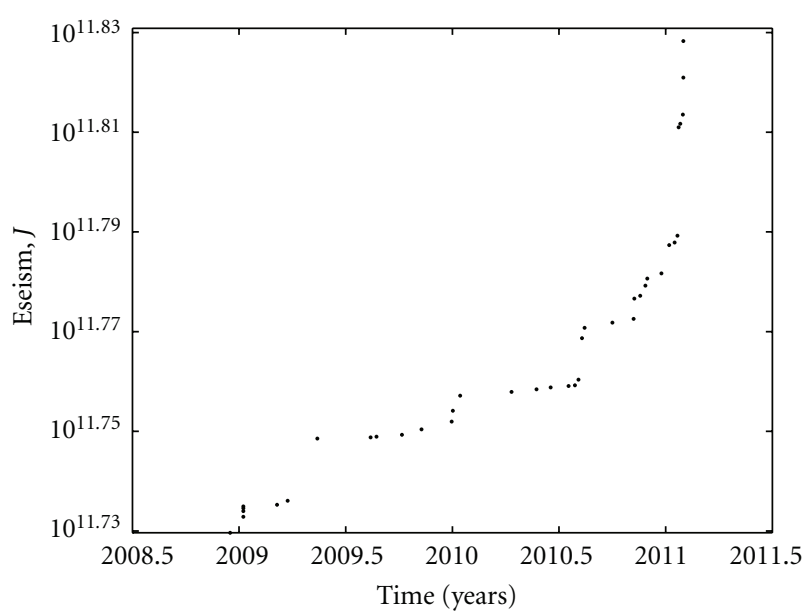

(b)

FIGURE 10: Cumulative graphs of a number of the events (a) and of released seismic energy (b) inside the area $48^{\circ}-49^{\circ} \mathrm{N}$ and $141.5^{\circ}-$ $143^{\circ} \mathrm{E}$. Vertical lines and figures show the moments of the Nevelsk (1), Gornozavodsk (2) earthquakes, and the strongest earthquake of February 24, 2007, $M=4.6$ (3) occurred in the pointed area.

in this time interval. A prominent nonlinear growth of a number of earthquakes preceded the first two events and coincides with the moment of the third event occurring in the Poyasok Isthmus area. The last case is an example of a typical fore- and aftershock behavior. The first two cases of nonlinear increases in seismic activity with the subsequent seismic silence agree with the seismic behavior found in the distant vicinity of the strong earthquakes in [27]. In this work, an increase in seismic activity replaced by the seismic silence was found in a distant vicinity of generalized strong earthquake with approaching the strong earthquake occurrence moment.

A nonlinear growth of a number of earthquakes takes place in the Poyasok Isthmus area also since 2010. Besides, the nonlinear growth of released seismic energy takes place here since the middle of 2010 (Figure 10(b)).

It should be noted also that the intervals of time of nonlinear growth of a number of events and of released seismic energy (occurring in 2007-2008 and since the middle of 2010) correspond to the intervals of time of occurrence of events with higher $(>0.55)$ values of parameter of metastability $p$ in the Poyasok Isthmus area. Thus one can conclude that the seismic regime behavior taking place now in the Poyasok Isthmus area repeats the one that took place here before the strong Nevelsk earthquake and testifies for the increase of probability of a strong earthquake occurrence.

\section{Discussion}

Seismic regime is usually considered in terms of the SOCmodel. This model suggests the spontaneous evolution of dynamic system to a critical state. However, the physical mechanism of such evolution in the case of seismicity has not been suggested. It is also not clear how to explain the difference of seismically active and aseismic areas in terms of the SOC-model. The analogy between seismic regime and the second-order phase transitions also seems disputable. The principal feature of the second-order phase transitions is that the transformation goes without absorption (emission) of energy. In contrast to it, a huge explosion-like release of energy takes place during strong earthquakes.

The alternative model of the seismic regime as a set of episodes of avalanche-like relaxation of metastable sub-systems (SEM model) is suggested. In the case of seismicity the origin of metastable sub-systems is connected with storage of elastic energy. The discharge of accumulated elastic energy can be initiated by the excess of stress level [2] and/or by local temporal decrease of strength of geomaterial occurring in connection with processes of (fluid) metamorphic transformations [28-30].

In the simple variant of the SEM model (without memory of the medium), the geophysical medium is described by two parameters [9]. The first parameter characterizes a spatial hierarchy (scaling) of the medium; this parameter $r$ is easily identified with the coefficient of hierarchy according to Sadovsky [31]. The second parameter characterizes a degree of metastability of the medium; it is parameter $p$, a probability of continuation of the process of on-going avalanchelike relaxation of the stored energy. In assemblage, these two parameters define spatial-time change of the $b$-value.

The presence of two latent parameters specifying one empirically determining characteristic $b$-value gives place for choice. It seems natural to describe the spatial changeability by scaling parameter $r$ and a temporal changeability by parameter of metastability $p$. The spatial model of change of scaling parameter for Sakhalin $R(\varphi, \lambda)$ was obtained using the catalogue for 1905-2005; at this step parameter $p$ was suggested to be constant $p=0.5$. The model of time changeability of parameter of metastability $p(\varphi, \lambda, t)$ was obtained with the use of values $R(\varphi, \lambda)$ on the basis of the detailed $(M \geq 2)$ catalogue of seismicity of the south of Sakhalin for the time interval 06/07/2003-02/27/2011, when strong Gornozavodsk and Nevelsk earthquakes $\left(M_{W} 5.6\right.$ and $\left.M_{W} 6.2\right)$ had occurred.

As a result of the estimate of the parameter of metastability $p(\varphi, \lambda, t)$, a few spatial-time groups of earthquakes with 
higher $p$ parameter values were revealed. In terms of the SEM model such increase corresponds to the growth of probability of the strong earthquake occurrence. One of the groups, observed before the Nevelsk earthquake, corresponds to the causative fault of this earthquake. After the Nevelsk earthquake, occurrence of the higher values of parameter $p$ was not observed for a year. Then such earthquakes appeared again in the area of the Poyasok Isthmus, as it was before the Nevelsk earthquake. Since March 2010 in this area, the shocks with still higher $(>0.6)$ values of the parameter $p$ have appeared. It can be suggested that such behavior testifies for an increase of the probability of a strong earthquake origin in the south of Sakhalin.

The results of parameterization of the seismic regime in the framework of the SEM model complement the results received previously from the examination of the seismic gaps. According to these results, the gap along the western coast of the South Sakhalin was only partly (in its southern part up to the latitude $47^{\circ} \mathrm{N}$ ) closed as a result of the Nevelsk earthquake (Figure 5). At present, it is not clear whether the finding of increase in seismic activity in the Poyasok Isthmus area shows a higher probability of the origin of a stronger earthquake in this area or a stronger earthquake can occur in the larger area corresponding to the seismic gap along the western coast of the Sakhalin Island. If the period of accelerated growth of seismic activity changes by the period of seismic silence (as it was just before the Nevelsk and Gornozavodsk earthquakes), it can be an indicator of a stronger and more remote earthquake.

Note that the revealed features in the behavior of parameters of the SEM model could be explained in terms of change of $b$-value. In this case, the discussed anomaly in parameter $p$ increase in the vicinity of strong Nevelsk earthquake corresponds to the well-known tendency of decrease in $b$-value in vicinity of strong earthquakes. But the used approach of the SEM model has some advantage for the interpretation because of the more clearly physical sense of the parameters of the SEM model.

\section{Conclusion}

Seismic regime is usually considered as an example of the regime of self-organizing criticality (SOC-conception). The alternative SEM model treats the seismic regime as an assemblage of random episodes of avalanche-like relaxation, taking place at a set of uniform metastable sub-systems. The SEM model in its simple form without memory of the system is defined by two parameters, characterizing scaling in spatial structure of the Earth's crust and the degree of metastability of the geophysical medium. This model is used for the description of seismic regime of the south of Sakhalin Island. The models of spatial changeability of the scaling parameter and temporal changeability of the parameter of metastability are constructed. The anomalous growth of the parameter of metastability preceded the occurrence of the Gornozavodsk and Nevelsk earthquakes. At the present time, the anomalously high (and growing over time) values of this parameter are observed in the area of the Poyasok Isthmus (in the vicinity of latitude $48^{\circ} \mathrm{N}$ ). Clear nonlinear growth of the flow of a number of seismic events and of seismic energy is noticeable in this area before the Gornozavodsk and Nevelsk earthquakes and after 2009.

\section{Acknowledgments}

This paper was supported by the Russian Foundation for Basic Research, Grant no. 11-05-00663, and the European Grant FP7 no. 262005 SEMEP.

\section{References}

[1] P. Bak, C. Tang, and K. Wiesenfeld, "Self-organized criticality," Physical Review A, vol. 38, no. 1, pp. 364-374, 1988.

[2] G. A. Sobolev and A. V. Ponomarev, Physics of Earthquakes and Precursors, Nauka, Moscow, Russia, 2003.

[3] D. L. Turcotte, "Seismicity and self-organized criticality," Physics of the Earth and Planetary Interiors, vol. 111, no. 3-4, pp. 275-293, 1999.

[4] Y. Ben-Zion, "Collective behavior of earthquakes and faults: continuum-discrete transitions, progressive evolutionary changes, and different dynamic regimes," Reviews of Geophysics, vol. 46, no. 4, Article ID RG4006, 2008.

[5] Y. Ogata, "Statistical models for earthquake occurrence and residual analysis for point processes," Journal of the American Statistical Association, vol. 83, pp. 9-27, 1988.

[6] Y. Ogata, "Space-time point-process models for earthquake occurrences," Annals of the Institute of Statistical Mathematics, vol. 50, no. 2, pp. 379-402, 1998.

[7] P. A. Reasenberg and L. M. Jones, "Earthquake hazard after a mainshock in California," Science, vol. 243, no. 4895, pp. 1173-1176, 1989.

[8] V. Pisarenko and M. Rodkin, "Heavy-tailed distributions in disaster analysis," Advances in Natural and Technological Hazards Research, vol. 30, 2010.

[9] M. V. Rodkin, "Alternative to SOC concept—model of seismic regime as a set of episodes of random avalanche-like releases occurring on a set of metastable subsystems," Izvestiya, Physics of the Solid Earth, vol. 47, no. 11, pp. 966-973, 2011.

[10] B. B. Mandelbrot, The Fractal Geometry of Nature, W.H. Freeman and Company, 1982.

[11] M. V. Rodkin, V. F. Pisarenko, and T. A. Rukavishnikova, "Parameterization of regime of rare strong catastrophesevents," Geoecology, no. 2, pp. 164-172, 2007 (Russian).

[12] Sornette D., Critical Phenomena in Natural Sciences, Chaos, Fractals, Self-organization and Disorder: Concepts and Tools, Springer Series in Synergetics, Heidelberg, Germany, 2nd edition, 2004.

[13] T. Utsu, "A method for determining the value of $b$ in a formula $\log n=a-b m$ showing the magnitude-frequency relation for earthquakes," Geophysical bulletin of Hokkaido University, vol. 13, pp. 99-103, 1965.

[14] D. Wei and T. Seno, "Determination of the Amuruan plate motion," in Mantle Dynamics and Plate Interactions in East Asia, vol. 27 of Geodynamics Series, p. 419, AGU, Washington, DC, USA, 1998.

[15] B. V. Levin, Ch. U. Kim, and I. N. Tikhonov, "The Gornozavodsk earthquake of August 17(18), 2006, in the south of Sakhalin Island," Russian Journal of Pacific Geology, vol. 1, no. 2, pp. 194-199, 2007. 
[16] I. N. Tikhonov and Ch. U. Kim, "Confirmed prediction of the 2 August 2007 MW 6.2 Nevelsk earthquake (Sakhalin Island, Russia)," Tectonophysics, vol. 485, no. 1-4, pp. 85-93, 2010.

[17] A. I. Kozhurin and M. I. Streltsov, "Seismotectonic consequences of the May 28, 1995 Northern Sakhalin Earthquake," Russia's Federal System of Seismological Networks and Earthquake Prediction. Information and Analytical Bulletin. The Neftegorsk Earthquake of May 27(28), 1995, pp. 95-100, 1995.

[18] T. Shimamoto, M. Watanabe, Suzuki et al., "Surface faults and damage associated with the 1995 Neftegorsk earthquake," The Journal of the Geological Society of Japan, vol. 102, no. 10, pp. 894-907, 1996.

[19] M. I. Streltsov, The May 27(28),1995 Neftegorsk Earthquake on Sakhalin Island, Yanus-K, Moscow, Russia, 2005.

[20] L. N. Poplavskaya, A. I. Ivaschenko, L. S. Oskorbin et al., Regional catalog of Sakhalin Earthquakes 1905-2005, Institute of Marine Geology and Geophysics Far Eastern Branch RAS, Yuzhno-Sakhalinsk, Russia, 2006.

[21] S. M. Saprygin, V. E. Kononov, and V. N. Senachin, "Horizontal motions and plate boundaries in Sakhalin and Hokkaido," Doklady Earth Sciences, vol. 398, no. 7, pp. 1043-1046, 2004.

[22] I. N. Tikhonov, Methods of Earthquake Catalog Analysis for Purposes of Intermediate- and Short-Term Prediction of Large Seismic Events, Institute of Marine Geology and Geophysics Far Eastern Branch RAS, Vladivostok, Russia, 2006.

[23] K. Mogi, Earthquake Prediction, Academic Press (Harcourt Brace Jovanovich, Publishers), New York, NY, USA, 1985.

[24] B. V. Levin, Ed., Catalog of Earthquakes of the South Sakhalin Area since 2000 until 2010 years, Vladivostok, Russia, 2011.

[25] S. M. Saprygin, "Detailed seismic zoning of Sakhalin," Russian Journal of Pacific Geology, vol. 2, no. 2, pp. 158-164, 2008 (Russian).

[26] N. A. Bogdanov, Ed., Tectonic Map of the Sea of Okhotsk Region in 1:2500000 Scale, ILOVM RAS, Moscow, Russia, 2000.

[27] M. V. Rodkin, "Seismicity in the generalized vicinity of large earthquakes," Journal of Volcanology and Seismology, vol. 2, no. 6, pp. 435-445, 2008.

[28] V. A. Kalinin, M. V. Rodkin, and I. S. Tomashevskaya, Geodynamic Effects of Physicochemical Transformations in a Solid Medium, Nauka, Moscow, Russia, 1989.

[29] M. V. Rodkin, A. D. Gvishiani, and L. M. Labuntsova, "Models of generation of power laws of distribution in the processes of seismicity and in formation of oil fields and ore deposits," Russian Journal of Earth Sciences, vol. 10, no. 5, 2008.

[30] D. Sornette, "Earthquakes: from chemical alteration to mechanical rupture," Physics Report, vol. 313, no. 5, pp. 237-291, 1999.

[31] M. A. Sadovskii, Ed., Discrete Properties of Geophysical Media, Nauka, Moscow, Russia, 1989. 

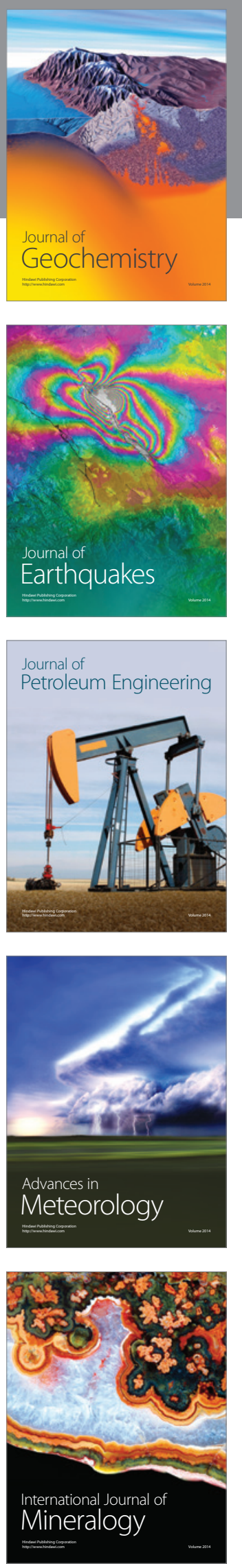
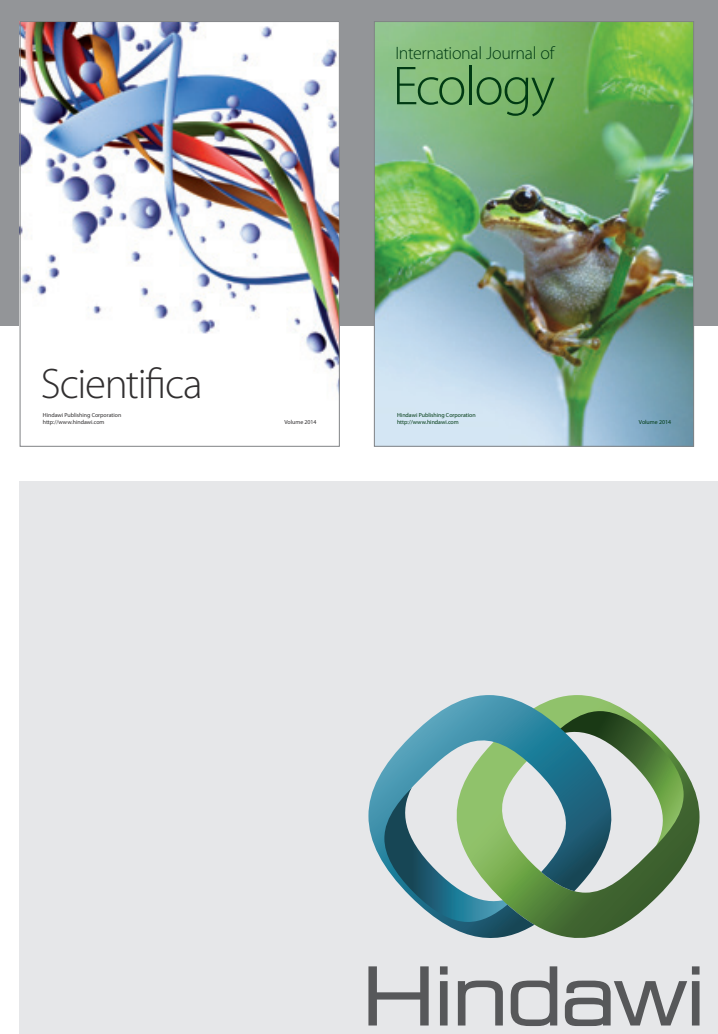

Submit your manuscripts at http://www.hindawi.com
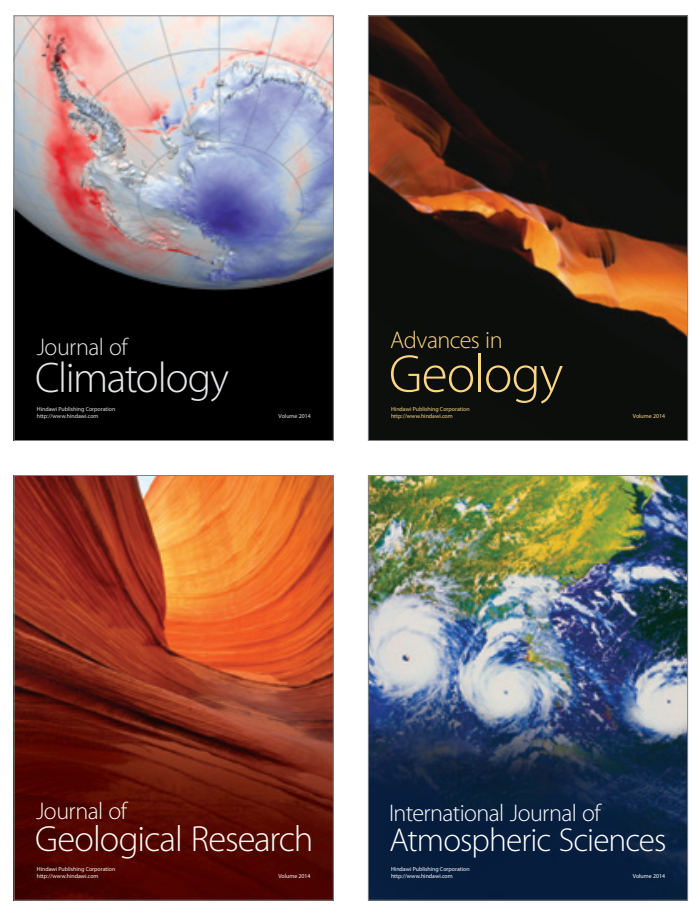
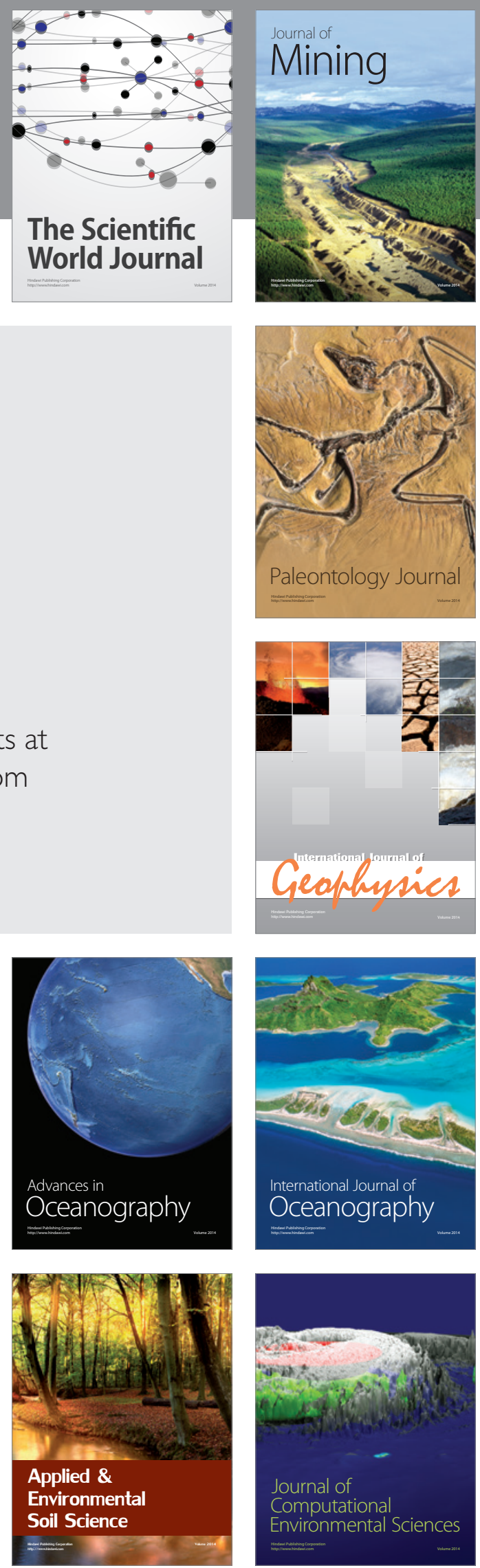